\title{
On the Electronic Spectrum of the Diatomic Molecular Ion
}

\author{
P. Aventini and R. Seiler ${ }^{\star}$ \\ Institut für Theoretische Physik, Freie Universität Berlin, Berlin
}

Received July 20, 1974

\begin{abstract}
Discrete eigenvalues of the electronic Hamiltonian with dilatation analytic potentials for ionized diatomic molecules are shown to depend smoothly on the distance $r$ of the nuclei for $r$ finite and non vanishing. Furthermore the spectrum is analyzed at $r=0$ and $r=\infty$ and the eigenvalues turn out to be continuous.
\end{abstract}

\section{Introduction}

In quantum theory of molecules one of the most important tools is the method of Born and Oppenheimer [1]. Prerequisites for a successful application of the method are spectral properties of the so called electronic Hamiltonian. The analysis of those is the subject of this note and is a part of a general study of the method of Born-Oppenheimer done in collaboration with Combes, Duclos and Grossmann $[2,3]$.

The problem discussed by Born and Oppenheimer is the following: Consider the Hamiltonian for a system of $N$ non relativistic particles, $S$ of them with mass $M$ and $L=N-S$ with mass $m$,

$$
\mathbb{H}=\frac{1}{2 M} \sum_{1}^{S} p_{s}^{2}+\frac{1}{2 m} \sum_{1}^{L} q_{e}^{2}+W\left(x_{1} \ldots x_{S}, y_{1} \ldots y_{L}\right) .
$$

Think of $M$ and $m$ being the mass of nuclei and electrons respectively. Then the mass ratio $\kappa^{4}=m / M$ is small and the problem arises to formulate a perturbation theory in $\kappa$ useful to get information about spectrum and eigenfunctions of $\mathbb{H}$. Such a perturbation theory is obviously singular because the small parameter multiplies the differential operator $\Sigma p_{s}^{2}$ which can not be expected to be in any sense small compared to the rest of $\mathrm{H}$. In their paper they give a formal but physically well motivated and successful account of such a perturbation theory. It turns out [3] that the problem can be cast in the frame of singular perturbation theory [4]. Here we will discuss the so to say zero order problem and analyse the spectrum of the electronic Hamiltonian $H_{e l}\left(x_{1} \ldots x_{s}\right)$ which is gotten from IH by putting $M=\infty$ or $\kappa=0$.

To concentrate on the relevant part of the problem let us consider just the Hamiltonian for an ionized diatomic colecule

$$
\underset{\sim}{H}=\sum_{i=1}^{2} \frac{1}{2 M_{i}} p_{i}^{2}+V_{i}\left(x_{i}-y_{1}\right)+\frac{1}{2 m} q_{1}^{2}+V_{3}\left(x_{1}-x_{2}\right) \text {. }
$$

\footnotetext{
* Supported in Part by DFG contract No.: Se 287/1.
} 
Introducing Jacobi coordinates and separating of the kinetic part of the center of mass motion, $\underset{\sim}{H}$ transforms into

$$
\begin{aligned}
H= & -\frac{1}{2 m} \kappa^{4} \cdot r \cdot \Delta_{x}-\frac{1}{2 m}\left(1+\kappa^{4}(1-1 / r)\right) \Delta_{y}+V_{1}(y+1 / r x) \\
& +V_{2}(y-(1-1 / r) x)+V_{3}(x) \\
x= & x_{1}-x_{2} \\
y= & \left(\Sigma M_{i} x_{i} / \Sigma M_{i}\right)-y_{1} \\
r= & M_{1} / M_{2}+1 .
\end{aligned}
$$

Setting $\kappa=0$ we get the electronic Hamiltonian which depends parametrically on the configuration $x$ of the nuclei

$$
H_{e l}(x)=-\frac{1}{2 m} \Delta_{y}+V_{1}(y+1 / r x)+V_{2}(y-(1-1 / r) x)+V_{3}(x) .
$$

Here $H(x)$ is considered as an operator on $L^{2}\left(d^{3} y\right)$. The last term in the definition of $H_{e l}(x)$ acts trivially on $L^{2}\left(d^{3} y\right)$. Hence we are left with the analysis of the operator

$$
H(x)=H_{0}+V_{1}(y)+V_{2}(y-x), \quad H_{0}=-\frac{1}{2 m} \Delta_{y}
$$

unitarily equivalent to the nontrivial part of $H_{e l}(x)$ :

$$
H(x)=T(1 / r x)\left\{H_{e l}(x)-V_{3}(x)\right\} T(-1 / r x)
$$

where $T(a)$ is the natural unitary representation of the group of translations on $L^{2}\left(d^{3} y\right)$.

The spectral properties of $H(x)$ will be discussed under the general assumption that $V_{l}, l=1,2$, are dilatation analytic (d.a.) potentials [5]. In a slight modification of the original concept we call $V$ a d.a. potential if the following conditions are satisfied:

i) $V\left(1+H_{0}\right)^{-1}$ is compact,

ii) $D(\lambda) V\left(1+H_{0}\right)^{-1} D(\lambda)^{-1}, \lambda \in R_{+}^{3}$, has an analytic extension as bounded operator on $L^{2}\left(d^{3} y\right)$ from $R_{+}^{3}$ into the sector

for a $\phi>0$.

$$
S_{\phi}=\left\{\lambda \in \mathbb{C}^{3}\left|\operatorname{Re} \lambda_{l}>0,\right| \arg \lambda_{l} \mid<\phi, l=1,2,3\right\}
$$

Here $D(\lambda)$ is the natural unitary representation of the dilatation group $R_{+}^{3}$ on $L^{2}\left(d^{3} y\right)^{1}$ :

$$
\begin{aligned}
(D(\lambda) f)(y) & =\Pi \lambda_{l}^{\frac{1}{2}} f(\lambda y) \\
\lambda y & =\left(\lambda_{1} y_{1}, \lambda_{2} y_{2}, \lambda_{3} y_{3}\right) .
\end{aligned}
$$

The Yukawa and the Coulomb potential are dilatation analytic. We will avoid throughout the paper to use methods not generalizable to the case of many particle systems. This generalization and the analysis of the eigenfunctions of $H(x)$ will be the subject of a separate publication.

1 Since the elements of $R_{+}^{3}$ have components strictly positive there is no problem with the definition of the square root. If more general canonical transformations on phase space are considered this situation might change [10]. 
Computer calculations have been very helpful for getting ideas about the general behaviour of the discrete spectrum of $H(x)$ [6]. For the case of the ionized Hydrogen molecule, where $V_{1}(y)=V_{2}(y)=-\frac{e^{2}}{|y|}, e$ denoting the electric charge, one finds the following results: The discrete eigenvalues of $H(x)$ depend smoothly on $x$ for $x \neq 0$. In fact they depend only on $r=|x|$. For $r$ approaching zero they converge to eigenvalues of the Coulomb problem with charge $2 e$. The lowest eigenvalue $\mu(x)$ is monotonically increasing with $r$. For $r=0$ it has the value -4 in units of Rydberg ( $\mathrm{Ry}=\frac{1}{2} m e$ ) and approaches -1 for $r \rightarrow \infty$. It never intersects any other discrete eigenvalue. This and the monotonicity property do not hold for some other eigenvalues. The eigenvalues of $H(x=0)$ are degenerate. The degeneracy is reduced for $x \neq 0$. A similar situation seems to hold at $r=\infty$.

Let us now outline the organization of the article and summarize at the same time the results. In the first chapter local properties of the spectrum of $H(x)$ are analyzed.

It is shown that the discrete eigenvalues can be indexed such that they are continuous in $x \in R^{3}$. Furthermore there exists a possibly different choice of indexing the discrete eigenvalues such that they are real analytic in every variable separatly, for $x \in R^{3} \backslash\{0\}$. The first statement is a consequence of norm resolvent continuity ( $n-r$ continuity) of $H(x), x \in R^{3}$.

The second one is very much linked to dilatation analyticity of the potentials. For $x \rightarrow \infty H(x)$ converges in the strong resolvent sense $(s-r$ convergence) to $H_{1}(\infty)$

$$
H_{l}(\infty)=H_{0}+V_{l}, \quad l=1,2 .
$$

It turns out that eigenvalues of $H_{1}(\infty)$ are only stable in the sense of Kato [4] iff they do not belong to the spectrum of $H_{2}(\infty)$. An analoguous statement holds for the convergence of $H^{\prime}(x)=T(-x) H(x) T(x)$. $H^{\prime}(x)$ converges in the $s-r$ sense to $H_{2}(\infty)$. The eigenvalues of $H_{2}(\infty)$ not in the spectrum of $H_{1}(\infty)$ are stable. In a second chapter we discuss global aspects of the spectrum. Results on global analyticity are demonstrated and bounds on the discrete spectrum are given. Some statements can be sharpened for the case of Coulomb or Yukawa interaction. In particular, the eigenvalues are differentiable at $x=0$.

Throughout the whole article we always suppose the potentials to be dilatation analytic. In anouncing the lemmas we will not restate that assumption again.

\section{Chapter 1: Local Properties of the Spectrum of $\mathbf{H}(\mathbf{x})$}

In this first chapter we will mainly prove some results on the resolvents of $H(x)$ and $H(\lambda, x)$ (to be defined later). This will allow us to apply standard arguments of perturbation theory to get information on local regularity properties of the discrete eigenvalues $\mu(x)$ of $H(x)$ as a function of $x$.

The operator $H(x)$ is selfadjoint with domain $D(H(x))=D\left(H_{0}\right)$ independent of $x \in R^{3}$. This is due to the general hypothesis mentioned in the introduction, that $V_{1}$ and $V_{2}$ are dilatation analytic potentials hence a fortiori $H_{0}$ compact. $V_{1}$ and $V_{2}$ are therefore Kato tiny [7]. Since $H_{0}$ is translationally invariant $T(x) V_{2} T(-x)$ is Kato tiny too. Hence $H(x)$ is the sum of $H_{0}$ and a Kato perturbation. 
Let $(z-H(x))^{-1}$ be the resolvent of $H(x)$. Then one has the following

Lemma 1. The resolvent of $H(x)$ is norm continuous in $x$ for $x \in R^{3}$.

Before entering into the proof let us introduce some standard notation: If a sequence of operators $\left\{T_{n}\right\}$ converges in norm (strongly) to $T$ for $n \rightarrow \infty$ we write $T_{n} \stackrel{n}{\rightarrow} T\left(T_{n} \stackrel{s}{\rightarrow} T\right)$ for $n \rightarrow \infty$. The resolvent set of an operator $T$ is denoted by $\varrho(T)$, the spectrum by $\sigma(T)$, the essential spectrum by $\sigma_{\text {ess }}(T)$ and the discrete spectrum by $\sigma_{\mathrm{d}}(T)$. The union of the spectra of $H_{1}(\infty)$ and $H_{2}(\infty)$ plays an essential role. Therefore we introduce the notation

$$
\begin{gathered}
\sigma_{\mathrm{d}}=\sigma_{\mathrm{d}}\left(H_{1}(\infty)\right) \cup \sigma_{\mathrm{d}}\left(H_{2}(\infty)\right) \\
\sigma=\sigma\left(H_{1}(\infty)\right) \cup \sigma\left(H_{2}(\infty)\right) .
\end{gathered}
$$

A function $T(\mathrm{z})$ of a complex variable $z$ with values in the set of closed linear operators on a Hilbert space $X$ is called analytic of Typ A at $z_{0}$ [4] if there exists a complex neighborhood $D$ of $z_{0}$ such that

i) $D(T(z)=D(T)$ is independent of $z$.

ii) $T(z) u$ is analytic in $D \forall u \in D(T)$.

If in addition $T(z)$ selfadjoint for $z \in D \cap R$ then $T(z)$ is a selfadjoint holomorphic family of linear operators. Discrete eigenvalues of $H(x)$ are denoted by $\mu(x)$, $x \in R^{3}$. If we want to look at them as a function of one variable only we use $\mu_{x}(\kappa)=\mu(\kappa x), \kappa \in R$. If $x$ is fixed and if there is no danger of confusion we write just $\mu(\kappa)$ for $\mu_{x}(\kappa)$.

Proof of Lemma 1. We show first norm continuity of $T(x) V_{2} T(-x)\left(z-H_{0}\right)^{-1}$ for $z \in \varrho\left(H_{0}\right)$. By the standard telescoping trick one gets

$$
\begin{aligned}
T(x) V_{2} T(-x)\left(z-H_{0}\right)^{-1}= & T(x)\left[V_{2}\left(z-H_{0}\right)^{-1} T(-x)-V_{2}\left(z-H_{0}\right)^{-1}\right] \\
& +T(x) V_{2}\left(z-H_{0}\right)^{-1} .
\end{aligned}
$$

Since $T(x) \stackrel{s}{\rightarrow} 1$ for $x \rightarrow 0$ and $V_{2}\left(z-H_{0}\right)^{-1}$ compact the second term of (9) converges in norm to $V_{2}\left(z-H_{0}\right)^{-1}$, (Theorem A). For the same reason the expression in the bracket converges in norm to zero and because $T(x)$ is unitary the first term of (9) converges in norm to zero.

Now we can prove the statement of the lemma. Consider the Neumann series which converges for $z$ sufficiently negative (this is generally true for perturbation of $H_{0}$ which are Kato tiny, Lemma 4)

$$
(z-H(x))^{-1}=\left(z-H_{0}\right)^{-1} \sum_{n=0}^{\infty}\left(\left(V_{1}+T(x) V_{2} T(-x)\right)\left(z-H_{0}\right)^{-1}\right)^{n} .
$$

Since every term of the sum is norm continuous at $x=0$ and the convergence of the sum is uniform in $x$ the resolvent is norm continuous at $x=0$. The generalization to $x \in R^{3}$ and $z \in e(H(x))$ arbitrary goes along standard lines and will be omitted.

The norm continuity of the resolvent $(z-H(x))^{-1}$ has the following consequence for the spectrum of $H(x)$ :

Theorem 1. Let $V_{1}$ and $V_{2}$ be d.a. potentials and $\mu\left(x_{0}\right) \in \sigma_{\mathrm{d}}\left(H\left(x_{0}\right)\right)$ for $x_{0} \in R^{3}$ where $H(x)$ is defined by (5). Then $\mu(x)$ is continuous in $x$ at $x=x_{0}{ }^{2}$.

\footnotetext{
${ }^{2}$ Here and in the following such statements should always be understood in the following sense: There exists a labelling of the eigenvalues such that $\mu(x)$ is continuous at $x=x_{0}$.
} 
Proof. The statement follows essentially from Lemma 1. The proof is a slight generalization of a standard argument in the theory of operator valued functions of one to the case of several variables. We will not repeat it and refer to $[4, \mathrm{p} .107]$. It is well known however that just this generalization is in general not trivial at all.

Notice that in Theorem 1 nothing is said about the possibility of continuing $\mu(x)$ into a larger region containing $x_{0}$. The statement is purely local. Theorem 1 can also be proven by the following reasoning. Lemma 1 implies the stability of the spectrum [4, p. 437]. This however implies continuity of discrete eigenvalues.

To get more information on the smoothness property of discrete eigenvalues we introduce the family $\{H(\lambda, x)\}$ of selfadjoint operators:

$$
H(\lambda, x)=D(\lambda) H(\lambda x) D^{-1}(\lambda), \quad \lambda \in R_{+}^{3} .
$$

Thereby we used the notation introduced previously (7). Since $D(\lambda)$ is unitary the spectra of $H(\lambda x)$ and $H(\lambda, x)$ are identical

$$
\sigma(H(\lambda x))=\sigma(H(\lambda, x))
$$

$H(\lambda, x)$ has the following remarkable property

Lemma 2. The operators $H(\lambda, x), \lambda \in S_{\phi}$ are a holomorphic family of selfadjoint operators of typ $A$.

Remark. $H(\lambda, x)$ is up to now only defined for $\lambda \in R_{+}^{3}$. The generalization is given in the proof below.

Proof 1. $H(\lambda, x)$ is selfadjoint with domain $D\left(H_{0}\right)$ for $\lambda$ real: We know that $H(\lambda x)$ has this property and it remains to show that $D\left(H_{0}\right)$ is invariant under $D(\lambda)$, a fact which follows easily from the definition of $D(\lambda)$.

2. $H(\lambda, x)$ with domain $D\left(H_{0}\right)$ is closed for every $\lambda \in D: H(\lambda, x)$ is defined on $D\left(H_{0}\right)$ as follows. For $u \in D\left(H_{0}\right)$ there exists a $v$ such that $u=\left(z-H_{0}\right)^{-1} v$ if $z$ is in the resolvent set of $H_{0}$. Since $V_{1}$ and $V_{2}$ are dilatation analytic $H(\lambda, x) u$ can be defined by

$$
\begin{aligned}
H(\lambda, x) u= & \lambda^{-2} H_{0}\left(z-H_{0}\right)^{-1} v+D(\lambda) V_{1} D^{-1}(\lambda)\left(z-H_{0}\right)^{-1} v \\
& +T(x) D(\lambda) V_{2} D(\lambda)^{-1}\left(z-H_{0}\right)^{-1} T(-x) v .
\end{aligned}
$$

Due to the fact that $D(\lambda) V_{1} D^{-1}(\lambda)$ and $T(x) D(\lambda) V_{2} D^{-1}(\lambda) T(-x)$ are $H_{0^{-}}$ compact for $\lambda \in D$ they are $H_{0}$ bounded with bound arbitrarily small [7, Theorem A 1]. A stability property of closed operators under such perturbation implies that $H(\lambda, x)$ is closed [4, p. 190].

3. $H(\lambda, x)$ is analytic in $\lambda$ for $\lambda \in D$ : It is enough to show existence of $H(\lambda, x)$ $\left(z-H_{0}\right)^{-1}$ as an analytic function of $\lambda \in D$ with values in the set of bounded operators. This however is an immediate consequence of dilatation analyticity and Eq. (13).

Since $H(\lambda x)$ and $H(\lambda, x)$ are unitarily equivalent for $\lambda$ real the previous lemma leads to the statement of

Theorem 2. Let $V_{1}$ and $V_{2}$ be d.a. potentials. Then for every component of $x$, say for instance $x^{1} \in R$, there exists a labelling of the discrete eigenvalues $\mu(x)$ $\in \sigma_{\mathrm{d}}(H(x))$ such that they are real analytic in $x^{1}$ for $x \in R^{3} \backslash\{0\}$. If $\mu(x)$ is nondegenerate then $\mu(x)$ is real analytic in all the variables together. 
Remarks. 1. Notice that here also nothing is claimed about the global domain of analyticity. We will have to say something on this problem in the following chapter (Theorem 8).

2. The fact that $\mu(x)$ is only analytic in every variable separately is the origin of some technical troubles. To avoid them we will discuss mainly functions of one variable $\kappa \in R$,

$$
\mu_{x}(\kappa)=\mu(\kappa x), \quad x \in R^{3} \text { fixed },
$$

if we are to deal with global aspects of discrete eigenvalues. It is readaly seen that $\mu_{x}(\kappa)$ is real analytic in $\kappa \in R \backslash\{0\}$ under the conditions of the above theorem.

3. The discrete eigenvalues of $H(x)$ can be considered as functions of $r=|x|$ only. This is a particularity of diatomic molecular ions. The eigenvalues are real analytic in $r \in R_{+}$due to the Theorem 2 .

4. The reader might be astonished by the statement of real analyticity in every component. The following two comments might clarify the situation:

a) The following classical example shows the problems of perturbation theory for holomorphic operator-valued functions of several variables. Let $\tilde{p}$ be the $2 \times 2$ matrix

$$
\tilde{p}=\sum_{l=1}^{2} p_{l} \sigma_{l}=\left(\begin{array}{ll}
0 & p_{1}-i p_{2} \\
p_{1}+i p_{2} & 0
\end{array}\right)
$$

holomorphic in $p \in C^{2}$. The $\sigma_{l}$ 's are the Pauli matrices. $\tilde{p}$ has the special representation

$$
\tilde{p}=|p|\left(P_{+}(p)-P_{-}(p)\right), \quad P_{ \pm}(p)=\frac{1}{2}(1 \pm \tilde{P} /|p|) .
$$

A natural choice of indices for the eigenvalues is the following:

$$
\mu_{\frac{1}{2}}(p)= \pm \sqrt{ } \Sigma p_{l}^{2} .
$$

The $\mu$ 's are continuous but not real analytic in every component of $p$. There is another choice

$$
\begin{aligned}
v_{1}(p) & =\mu_{1}(p) \text { for } & p_{2} \neq 0 \\
& =p_{1} \quad \text { for } & p_{2}=0 \\
v_{2}(p) & =-v_{1}(p) . &
\end{aligned}
$$

For this choice of the labelling the eigenvalues are real analytic in $p_{1} \in R$ but neither continuous in both variables nor real analytic in $p_{2} \in R$.

4. Finally we mention that some special examples to be considered later indicate that $\mu(x)$ is in general not analytic at $x=0$ but only continuous (Theorem 1).

Proof of Theorem 2. 1. $\mu(\lambda) \in \sigma_{\mathrm{d}}(H(\lambda x))$ is real analytic in every component: If two components of $\lambda \in R^{3}$ are fixed, the operators $H(\lambda, x)$ are analytic in the last variable (Lemma 2). Now the statement follows by well known arguments on perturbation theory in one variable $[4, \mathrm{p} .386]$.

2. If $\mu(\lambda)$ is non degenerate then it is real analytic in $\lambda \in R^{3}$ : The projector of $\mu(\lambda)$,

$$
P(\lambda)=\frac{1}{2 \pi i} \int_{\Gamma} d z(z-H(\lambda, x))^{-1}
$$


is analytic in a complex neighborhood of $\lambda$ and has dimension one. Due to the formula

$$
\mu(\lambda)=\text { Trace } H(\lambda, x) P(\lambda)
$$

this implies the statement.

3. The statement of Paragraphs 1 and 2 hold for $\mu(x) \in \sigma_{\mathrm{d}}(H(x))$ if $x_{l} \neq 0$ for $l=1,2,3$ : The mapping $\phi: \lambda \rightarrow \phi(\lambda), \phi(\lambda)_{l}=\lambda_{l} / x_{l}$, mapps a complex neighborhood of $e=(1,1,1)$ onto a complex neighborhood of $x \cdot \phi$ is analytic.

4. The statements of the first two paragraphs hold for $\mu(x) \in \sigma_{\mathrm{d}}(H(x))$ if $x \neq 0$ : We will reduce this case to the one discussed previously. There exists a rotation $R$ of $R^{3}$ such that $(R x)_{l} \neq 0 ; l=1,2,3$. Yet $H(x)$ is unitarily equivalent to the operator $H^{\prime}(x)$,

$$
\begin{aligned}
H^{\prime}(x) & =H_{0}+V_{1}^{\prime}+T(R x) V_{2}^{\prime} T(-R x) \\
V_{l}^{\prime}(z) & =V_{l}\left(R^{-1} z\right), \quad l=1,2 .
\end{aligned}
$$

$H(x)$ and $H^{\prime}(x)$ have the same spectrum and $H^{\prime}(x)$ falls in the same class of Hamiltonians if we can show that $V_{l}^{\prime}$ is dilatation analytic together with $V_{l}$. This however will not be true for $R$ arbitrary. However if $R$ is chosen sufficiently close to $\mathbb{1}$ this is true as we will argue now.

The unitary representation $U(R)$ of the rotation group

$$
(U(R) f)(y)=f\left(R^{-1} y\right)
$$

has the following commutation law with $D(\lambda)$ :

$$
\begin{aligned}
D(\lambda) U(R) & =\Pi\left(\lambda_{i} \lambda_{i}^{\prime-1}\right)^{\frac{1}{2}} U(R) D\left(\lambda^{\prime}\right) \\
T(R): \lambda \rightarrow \lambda^{\prime}, \lambda_{l} & =\sum_{k} R_{l k} \lambda_{k} R_{k l}^{-1} .
\end{aligned}
$$

In the following we suppress the index of $V_{l}$ and write just $V$ or $V^{\prime}=U(R) V U(R)^{-1}$. If $V$ is a d.a. potential the operator

$$
D(\lambda) V^{\prime}\left(z-H_{0}\right)^{-1} D(\lambda)^{-1}
$$

is compact for $\lambda$ real. This follows from (15) and the boundedness of $U(R)$,

$$
D(\lambda) V^{\prime}\left(z-H_{0}\right)^{-1} D(\lambda)^{-1}=U(R) D\left(\lambda^{\prime}\right) V\left(z-H_{0}\right)^{-1} D\left(\lambda^{\prime}\right)^{-1} U(R)^{-1} .
$$

Hence the first condition for $V^{\prime}$ to be a d.a. potential is met. Yet the analytic continuation of (16) into a Sector $S_{\phi^{\prime}}$ is possible if $R$ is not too large. This is again a consequence of (16) and the following obvious fact: Let $\phi^{\prime}<\phi$ then for a $\varepsilon>0$ every transformation $T(R)$ with $\|R-1\|<\varepsilon$ mapps $S_{\phi^{\prime}}$ into $S_{\phi}$.

Now we come to the discussion of the spectrum of $H(x)$ at $x=\infty$. The situation is quite complicated since $H(x)$ converges in the strong resolvent sense to $H_{1}(\infty)$ (Theorem 3) and not in the norm resolvent-sense (Remark 2 following Theorem 4). Not every point in $\sigma_{\mathrm{d}}\left(H_{1}(\infty)\right)$ is stable but the spectrum of $H(x)$ is continuous (Theorem 5 and 6).

The next theorem shows that the behaviour of $H(x)$ at $x=\infty$ can be put in the framework of singular perturbation theory:

Theorem 3. Let $V_{l}$ be d.a. potentials, $l=1$, 2. Then $H(x)$ converges to $H_{1}(\infty)$ in the strong resolvent sense. 
This theorem implies already that for any $\mu \in \sigma_{\mathrm{d}}\left(H_{1}(\infty)\right)$ and $(a, b)$ open interval in $R$ with $\mu \in(a, b)$ the intersection of $(a, b)$ with $\sigma_{\mathrm{d}}(H(x))$ is not empty for $x$ sufficiently large. This is due to standard theorems on operators converging in the $s-r$ sense [4, p. 472].

Proof of Theorem 3. 1. It is enough to show strong convergence to zero of $T(x) V_{2} T(-x)\left(z-H_{0}\right)^{-1}$ for $|x| \rightarrow \infty$ and $z$ non real: Consider the resolvent equation

$\left.z-H(x))^{-1}-\left(z-H_{1} \infty\right)\right)^{-1} u=(z-H(x))^{-1} T(x) V_{2} T(-x)\left(z-H_{1}(\infty)\right)^{-1} u$.

The right hand side has a norm smaller then $|\operatorname{Im} z|^{-1}$ times $\| T(x) V_{2} T(-x)$ $\left(z-H_{1}(\infty)\right)^{-1} u \|$. Yet $\left(z-H_{1}(\infty)\right)^{-1} u$ is in $D\left(H_{0}\right)$ hence there exists a $v$ such that

$$
\left(z-H_{1}(\infty)\right)^{-1} u=\left(z-H_{0}\right)^{-1} v .
$$

Therefore the right hand side of (17) is dominated in norm by

$$
|\operatorname{Im} z|^{-1}\left\|V_{2} T(-x)\left(z-H_{0}\right)^{-1} v\right\| .
$$

2. $V_{2} T(-x)\left(z-H_{0}\right)^{-1}$ converges strongly to zero for $|x| \rightarrow \infty$ and $z$ non real: By hypothesis $V_{2}\left(z-H_{0}\right)^{-1}$ is compact. It has therefore the standard representation [8, p. 204]

$$
V_{2}\left(z-H_{0}\right)^{-1}=\sum_{i=1}^{\infty} \lambda_{i} e_{i} \otimes f_{i}, \quad \lambda_{i} \geqq 0 .
$$

Consider now the expression

$$
\begin{aligned}
\left\|V_{2}\left(z-H_{0}\right)^{-1} T(-x) \phi\right\|^{2} & =\left\|\Sigma \lambda_{i} e_{i}\left(f_{i}, T(-x) \phi\right)\right\|^{2} \\
& =\Sigma \lambda^{2}{ }_{i}\left|\left(f_{i}, T(-x) \phi\right)\right|^{2}
\end{aligned}
$$

converging for every $x$ in particular for $x=0$. For every $\varepsilon>0$ independent of $x \in R^{3}$ there exists a $N(\varepsilon)$ such that

$$
\sum_{i>N} \lambda_{i}^{2}\left|\left(f_{i}, T(-x) \phi\right)\right|^{2}<\varepsilon / 2 .
$$

Now the first $N$ terms of (19) converge to zero each one separatly due to the lemma of Riemann and Lebesgue.

The next technical statement will be the main tool for the discussion of the spectrum of $H(x)$ at $x=\infty$. The proof will be given in Appendix B.

Lemma 3. Let $z \in \mathbb{C}$ be in $\varrho(H(x)) \cap \varrho\left(H_{1}(\infty)\right) \cap \varrho\left(H_{2}(\infty)\right)$ for $x$ in a neighborhood of infinity. Then the operator $E(x, z)$ defined by

$$
\begin{aligned}
(z-H(x))^{-1}-\left(z-H_{1}(\infty)\right)^{-1}= & T(x)\left(z-H_{2}(\infty)\right)^{-1} V_{2}\left(z-H_{0}\right)^{-1} T(-x) \\
& +(z-H(x))^{-1} E(x, z)
\end{aligned}
$$

is a bounded operator. The norm of $E$ can be estimated as follows: Consider $\varepsilon>0$ and a compact set $K \subset \mathbb{C}$ such that the distance $d(K, \sigma)>\varepsilon$.

Then there exists for every $\eta>0$ a $\delta(\eta, K)$ such that $\|E(x, z)\|<\eta$ for $z \in K$ and $\delta|x|>1$. 
Notice that $E(x, z)$ exists as a bounded operator for every $z \in e\left(H_{l}(\infty)\right), l=1,2$. This is a consequence of the following formula (see Appendix B):

$$
E(x, z)=\sum_{l=1}^{2} T(x) V_{3-l} T(-x)\left(z-H_{0}\right)^{-1} V_{l}\left(z-H_{l}(\infty)\right)^{-1} .
$$

The following statement shows that the spectrum of $H_{1}(\infty)$ has some aspects of stability at infinity:

Theorem 4. Let $V_{1}$ and $V_{2}$ be d.a. potentials and $K$ a compact set in $\mathbb{C}$ such that $K \cap \sigma=\emptyset$.

Then there exists a $\delta(K)>0$ such that $K C \varrho(H(x))$ for every $x$ with $|x| \delta>1$.

Proof. Since $K$ is compact there exists a $\delta$ such that $\|E(x, z)\|<\frac{1}{2}$ for $z \in K$ and $|x| \delta>1$. The following formula for the resolvent is well defined

$$
\begin{aligned}
(z-H(x))^{-1}= & \left\{\left(z-H_{1}(\infty)\right)^{-1}+T(x)\left(z-H_{2}(\infty)\right)^{-1} V_{2}\left(z-H_{0}\right)^{-1} T(-x)\right\} \\
& \cdot(1-E(x, z))^{-1}
\end{aligned}
$$

for $z \in K$ and $|x| \delta>1$.

Remarks. 1. Consider $\lambda \in \sigma_{\mathrm{d}}$ and $K=R\left(\delta_{1}, \delta_{2}\right)$

$$
R\left(\delta_{1}, \delta_{2}\right)=\left\{z \in \mathbb{C}\left|\delta_{1}<\right| \lambda-z \mid<\delta_{2}<\infty\right\} .
$$

Then $R\left(\delta_{1}, \delta_{2}\right) \subset \varrho(H(x))$ for $|x|$ sufficiently large. This property is stronger then the first condition of stability for the spectrum of $H(x)$ at $x=\infty$ [4, p. 437].

2. Formula (23) permits the definition of projectors

$$
P(x)=\frac{1}{2 \pi i} \int_{\Gamma} d z(z-H(x))^{-1}, \quad P_{l}(\infty)=\frac{1}{2 \pi i} \int_{\Gamma} d z\left(z-H_{l}(\infty)\right)^{-1} l=1,2
$$

where $\Gamma$ is the circle around $\lambda \in \sigma_{\mathrm{d}}$ with radius $\frac{1}{2}\left(\delta_{1}+\delta_{2}\right)$. From (21) one concludes

$$
P(x)-P_{1}(\infty)=T(x) P_{2}(\infty) V_{2}\left(\lambda-H_{0}\right)^{-1} T(-x)+\frac{1}{2 \pi i} \int_{\Gamma} d z(z-H(x))^{-1} E(x, z) .
$$

The last term can be estimated in norm

$$
\begin{aligned}
\left\|\frac{1}{2 \pi i} \int_{\Gamma} d z(z-H(x))^{-1} E(x, z)\right\| & <\operatorname{Max}_{z \in \Gamma}\left\|(z-H(x))^{-1}\right\|\|E(x, z)\| \\
& \leqq \frac{2}{\left|d_{1}-d_{2}\right|} \eta
\end{aligned}
$$

where $\eta$ can be made arbitrarily small according to Lemma 3 if only $|x|$ is big enough. This proves that $P(x)$ does not converge in norm to $P_{1}(\infty)$ but rather that

$$
\left\|P(x)-P_{1}(\infty)\right\| \rightarrow\|F(x)\|=\|F(0)\| \quad \text { for } \quad|x| \rightarrow \infty
$$

where we used the abreviation

$$
F(x)=T(x) P_{2}(\infty) V_{2}\left(\lambda-H_{0}\right)^{-1} T(-x) .
$$

Notice however that $F(x) \stackrel{s}{\rightarrow} 0$ for $|x| \rightarrow \infty$ and

$$
P(x)-F(x)-P_{1}(\infty) \stackrel{n}{\rightarrow} 0 \text { for }|x| \rightarrow \infty .
$$


For the spectrum of $H_{1}(\infty)$ to be stable it is necessary and sufficient that the projectors $P(x)$ converge to $P_{1}(\infty)$ in norm. This is in general not correct as it has been demonstrated above. However the following statement holds:

Theorem 5. Let $V_{1}$ and $V_{2}$ be d.a. potentials and let $\lambda \in \sigma_{\mathrm{d}}\left(H_{1}(\infty)\right)$ but not in $\sigma_{\mathrm{d}}\left(H_{2}(\infty)\right)$. Then $\eta$ is stable. Every other point of $\sigma_{\mathrm{d}}\left(H_{1}(\infty)\right)$ is unstable.

Remark. We knowalready that the first condition of stability is met for every $\lambda \in \sigma_{\mathrm{d}}\left(H_{1}(\infty)\right)$ (Remark 1, following Theorem 4). Hence instability arises only through violation of the second condition i.e.

$$
\operatorname{dim} P(x) \leqq \operatorname{dim} P_{1}(\infty),
$$

where $P(x)$ and $P_{1}(\infty)$ has been defined previously (24). Yet $P_{1}(\infty)$ is a projector on an eigenspace related to an eigenvalue of $H_{1}(\infty)$. Similarly $P(x)$ projects among others on an eigenspaces of $H(x)$ with an eigenvalue $\mu(x)$ converging to $\lambda$. There is good reason to assume the dimensionality of this eigenspace to be equal to the one of $P_{1}(\infty)$ for $|x|$ sufficiently large. Yet there is an eigenvalue $\mu^{\prime}(x)$ converging to $\lambda$, because $H^{\prime}(x)$ converges strongly to $H_{2}(\infty)$ and $\lambda \in \sigma_{\mathrm{d}}\left(H_{2}(\infty)\right)$. $\mu^{\prime}(x)$ is of course eigenvalue of $H(x)$ too. $P(x)$ projects by definition on the corresponding eigenspace too, for $|x|$ sufficiently large. It seem therefore reasonable that the dimensionality of $P_{1}(\infty)$ is not larger or equal then the one of $P(x)$. So much for motivation and interpretation of Theorem 5 .

Proof. It is readaly seen that in this case $F(x)=0$. This is so because $P_{2}(\infty)=0$ for $\Gamma$ sufficiently small. Yet the assertion follows from Eq. (25).

The above statement proves already that the limit points of $\sigma_{\mathrm{d}}(\mathrm{H}(\mathrm{x}))$ for $|x| \rightarrow \infty$ contain the points of $\sigma_{\mathrm{d}}\left(H_{1}(\infty)\right)$ not in $\sigma_{\mathrm{d}}\left(H_{2}(\infty)\right)$ since this is a direct consequence of stability of this part of the spectrum [4, p. 439].

More details on the spectrum of $H(x)$ at $x=\infty$ are given in the following

Theorem 6. Let $V_{1}$ and $V_{2}$ be d.a. potentials and $\mu(x) \in \sigma_{\mathrm{d}}(H(x))$ continuous in $x$ for $x \in R^{3}$ and $|x|>r$.

Then $\mu(x)$ is continuous at infinity and

$$
\mu=\lim _{|x| \rightarrow \infty} \mu(x) \in \sigma_{\mathrm{d}} \cup\{0\} .
$$

Remarks. 1. In the following chapter we will show that $\mu(x)$ is already well defined for $|x|>r$ if $r$ is sufficiently large (Corollary 2 of Theorem 8).

2. If $\lambda \in \sigma_{\mathrm{d}}$ then there exists $\mu(x) \in \sigma_{\mathrm{d}}(H(x))$ such that $\mu(x)$ continuous in $x$ for $|x|>r$ and $\lambda=\lim _{\kappa \rightarrow \infty} \mu(\kappa x)$. This is again a consequence of Theorem 6 and Corollary 2 of Theorem 8 .

Proof of Theorem 6. 1. The set $P$ of limit points of $\{\mu(x)|| x \mid>r\}$ is not empty: According to Theorem 7 the function $\mu(x)$ is bounded from above and below

$$
-M<\mu(x)<0 \text {. }
$$

Hence by the Bolzano-Weierstrass theorem there is at least one point in $P$.

2. There is only one point called $\mu \in P$ : Assume the existence of two points $\mu_{1}$ and $\mu_{2}$ in $P, \mu_{1} \neq \mu_{2}$. There exists a compact set $K \subset \mathbb{C}$ such that $\operatorname{Re} K \subset\left(\mu_{1}, \mu_{2}\right)$ and $K \cap \sigma=\operatorname{Re} K \cap \sigma=\emptyset$. Yet according to Theorem 4 there exists a $\delta>0$ such 
that $K \cap \sigma(H(x))=\emptyset$ for all $x$ with $|x| \delta>1$. Because of the continuity of $\mu(x)$ in $x$ this contradicts the assumption.

3. $\mu \in \sigma_{\mathrm{d}} \cup\{0\}$ : Inequality (27) forces $\mu$ to be in the closed interval $[-M, 0]$. If $\mu \notin \sigma_{\mathrm{d}} \cup\{0\}$ there is a compact set $K$ containing $\mu$ such that $\operatorname{Re} K \cap \sigma_{\mathrm{d}}=\emptyset$. According to Theorem 4 the spectrum of $H(x)$ does not interset $K$ for $|x|$ sufficiently large. This contradicts the assumption $\mu \in P$.

This concludes the analysis of local properties of $\sigma_{\mathrm{d}}(H(x))$. Of course we could have discussed equally well $H^{\prime}(x)=T(-x) H(x) T(x)$. The statements of this chapter apply to $H^{\prime}(x)$ if $V_{1}$ and $V_{2}$ are interchanged and $x$ is replaced by $-x$.

\section{Chapter II. Global Properties of the Spectrum}

In a first section bounds on $\mu(x) \in \sigma_{\mathrm{d}}(H(x))$ will be given. The next section concerns the global domain of real analyticity. The results are not fully satisfactory, because we have neither been able to give necessary and sufficient conditions for crossing eigenvalues $\left(\mu_{l}(x) \in \sigma_{\mathrm{d}}(H(x))\right), l=1,2, \mu_{1}\left(x_{0}\right)=\mu_{2}\left(x_{0}\right), \mu_{1}(x)$ $>\mu_{2}(x), \mu_{1}\left(x^{\prime}\right)<\mu_{2}\left(x^{\prime}\right)$ in a neighbourhood of $\left.x_{0}\right)$ nor for the absorption of discrete eigenvalues into the continuum. However some more precise results are known for $x$ in a neighborhood of infinity and for Coulomb and Yukawa potentials. This will be the topic of the last section of this chapter.

To get an upper bound on every $\mu(x) \in \sigma_{\mathrm{d}}(H(x))$ we make use of stability of the essential spectra

$$
\sigma_{\text {ess }}(H(x))=\sigma_{\text {ess }}\left(H_{0}\right)
$$

for $H_{0}$-compact perturbations. A lower bound is easily derived from

Lemma 4. [9]. Let $V$ be a Kato tiny perturbation of $H_{0}$. Then $V\left(z-H_{0}\right)^{-1} \stackrel{n}{\rightarrow} 0$ for $\operatorname{Re} z \rightarrow-\infty$.

Remark. The above statement is of course wellknown and we will give a proof only because it is short and for convenience of the reader.

Proof. Let $\varepsilon>0$. Then there exists a finite real number $b(\varepsilon)$ such that

$$
\|V \phi\|<\frac{1}{3} \varepsilon\left\|H_{0} \phi\right\|+b\|\phi\| \quad \text { for all } \phi \in D\left(H_{0}\right) .
$$

This implies for $z$ with $\operatorname{Re} z<0$

$$
\left\|V\left(z-H_{0}\right)^{-1} \psi\right\|<\frac{2}{3} \varepsilon\|\psi\| \mid+b(\varepsilon) \frac{1}{|z|}\|\psi\| .
$$

For $|z|>b(\varepsilon), \operatorname{Re} z<0$, the right hand side is smaller then $\varepsilon$.

Theorem 7. Let $V_{1}$ and $V_{2}$ be d.a. potentials. Then there exists a finite $M \in R$ such that $\mu_{\mathrm{d}}(H(x)) \subset[-M, 0]$.

Proof. From (28) one gets the upper bound. For the lower bound consider the Neuman series

which converges for

$$
(z-H(x))^{-1}=\left(z-H_{0}\right)^{-1} \sum_{n \geqq 0}\left\{\left(V_{1}+T(x) V_{2} T(-x)\right)\left(z-H_{0}\right)^{-1}\right\}^{n}
$$

$\left\|\left(V_{1}+T(x) V_{2} T(-x)\right)\left(z-H_{0}\right)^{-1}\right\|<\left\|. V_{1}\left(z-H_{0}\right)^{-1}\right\|+\left\|V_{2}\left(z-H_{0}\right)^{-1}\right\|<1$.

This is the case for $z$ sufficiently negative (Lemma 4). 
Global analyticity of $\mu_{x}(\kappa)$ can in general not be demonstrated. There are arguments which makes one believe that eigenvalues can get absorbed in the continuum for appropriate values of $\kappa$ ruining so global analyticity. However eigenvalues which are sufficiently below the threshold of $\sigma_{\text {ess }}(H(x))$ are globally analytic. This is the statement of

Theorem 8. Let $V_{1}$ and $V_{2}$ be d.a. potentials and $\mu_{x}(\kappa) \in \sigma_{\mathrm{d}}(H(\kappa x))$ real analytic at $\kappa=1$. Then the following alternative holds: Either $\mu_{x}(\kappa)$ gets absorbed into the continuum i.e. there exists a real $\kappa_{0} \neq 0$ such that $0=\lim _{\kappa \rightarrow \kappa_{0}} \mu(\kappa), \mu_{x}(\kappa)$ analytic in $\left[1, \kappa_{0}\right)$; or $\mu_{x}(\kappa)$ real analytic for $\kappa \in R_{+} \backslash\{0\}$.

Proof. Let $I=(a, b) \subset R$ be the domain of real analyticity of $\mu_{x}(\kappa)$. Assume furthermore a or $b$ to be finite and not zero. We will show that $\mu(a)$ respectively $\mu(b)$ vanishes. The proof will be given for one end point of $I$ only. Let a be finite and not zero. We denote by $P$ the set of limit points $\lim _{n \rightarrow \infty} \mu\left(\kappa_{n}\right), \kappa_{n} \in I, \kappa_{n} \rightarrow a$ for $n \rightarrow \infty$. An argument identical to the one used in Paragraphe 2 of the proof of Theorem 6 shows $P$ to contain one point only. From this follows the existence of a unique limit $\mu(a)$

$$
\mu(a)=\lim _{\kappa \rightarrow a} \mu(\kappa)
$$

If $\mu(a) \neq 0$ it is in $\sigma_{\mathrm{d}}(H(a))$. Therefore $\mu(\kappa)$ has to be analytic at $\kappa=a$ (Theorem 2). This contradicts the assumption that a is boundary point of the domain of analyticity.

From Theorem 8 one draws the following two conclusions:

Corollary 1. Consider a point $\mu \in \sigma_{\mathrm{d}}$. Then there exists $\mu(x) \in \sigma_{\mathrm{d}}(H(x))$ such that $\mu_{x}(\kappa)=\mu(\kappa x)$ is analytic in $\kappa>1$ and $\lim _{\kappa \rightarrow \infty} \mu_{x}(\kappa)=\mu$.

Proof. By Theorem 3 and Theorem 1.14 of Ref. [4, p. 431] every real neighborhood $U(\varepsilon)$ of $\mu$ has a non empty intersection with $\sigma_{\mathrm{d}}(H(x))$ for $|x|>r, r$ sufficiently large. Call $\mu_{+}\left(\mu_{-}\right)$the adjacent points of $\mu$ in $\sigma_{\mathrm{d}}$ larger (smaller) then $\mu$. Due to Theorem 4 there exists an $r$ such that $K \subset \varrho(H(x))$ for $|x|>r$ where $K$ denotes the set

$$
K=\left[\mu+\frac{2}{3}\left(\mu_{+}-\mu\right), \mu+\frac{1}{3}\left(\mu_{+}-\mu\right)\right] \cup\left[\mu-\frac{2}{3}\left(\mu-\mu_{-}\right), \mu-\frac{1}{3}\left(\mu-\mu_{-}\right)\right] .
$$

Chose now $\mu(x) \in \sigma_{\mathrm{d}}(H(x))$ such that $|x|>r$ and $\mu(x) \in U(\varepsilon)$. Then due to Theorem 8 $\mu_{x}(\kappa)=\mu(\kappa x)$ is analytic in $[1, \infty)$. It converges to $\mu$ for $k \rightarrow \infty$ (Theorem 6).

Corollary 2. Let $K \subset \mathbb{C}$ be a compact set with $K \cap \sigma=\emptyset$ and $\delta>0$ such that $K \subset \varrho(H(x))$ for $|x| \delta>1$. Then for a given $x$ with $|x| \delta>1$ every $\mu_{x}(\kappa)=\mu(\kappa x)$ $\in \sigma_{\mathrm{d}}(H(\kappa x))$ such that $\mu(x)<\operatorname{Re} K$ is not only analytic in $\kappa$ at $\kappa=1$ but has a real analytic extension into the interval $[1, \infty)$.

Proof. $\mu(\kappa)$ is analytic at $\kappa=1$. Due to the assumption and the continuity of its analytic extension $\mu(\kappa)<\operatorname{Re} K$ for every $\kappa>1$ in the domain of analyticity. Hence $\mu(\kappa)$ can not get absorbed in the continuum and the second case of the alternative (Theorem 8) holds.

This terminats the general analysis of the spectrum of $H(x)$ for arbitrary dilatation analytic potentials. In the last section we comment on the case of 
Yukawa and Coulomb potentials, i.e.

$$
V_{l}(y)=-\frac{g_{l}}{|y|} \cdot \exp -\alpha_{l}|y|, \quad g_{l} \in R, \alpha_{l} \geqq 0 .
$$

The discrete spectrum of $H_{l}(\infty)$ for $\alpha=0$ is of course well know

$$
\sigma_{\mathrm{d}}\left(H_{l}(\infty)\right)=\left\{-\frac{1}{n^{2}} \cdot \frac{m g_{l}^{2}}{2} \mid n \in Z_{+}\right\} .
$$

For $\alpha>0$ there is only a finite number of points in $\sigma_{\mathrm{d}}$. The lowest eigenvalue $\mu_{0}(x)$ of $H(x)$ is bounded from above

$$
\mu_{0}(x)<\operatorname{Min}\left(-\frac{1}{2} m g_{1}^{2},-\frac{1}{2} m g_{2}^{2}\right) .
$$

This is a consequence of

$$
H(x) \leqq H_{1}(\infty), \quad H^{\prime}(x)=T(x) H(x) T(-x) \leqq H_{2}(\infty),
$$

and the minimax principle $[4, \mathrm{p} .61]$. This bound is optimal because $\mu_{0}(x)$ reaches the bound for $|x| \rightarrow \infty$ (Theorem 6 ).

The discrete eigenvalues are functions of $r=|\mathrm{x}|$ only. They are analytic in $r \in(0, \infty)$ and continuous at the boundary. For the special case of the Coulomb of Yukawa potential they are differentiable as it will be shown now for the case $\alpha=0$.

Consider a discrete eigenvalue $\mu(r)$ and a corresponding eigenvector $\phi(x)$.

$$
\mu(r)=(\phi(x), H(x) \phi(x)) .
$$

For the derivative one gets

$$
\mu^{\prime}(r)=g_{2} \int d^{3} y\left|\phi\left(x_{0} y\right)\right|^{2} \frac{\left(r-y^{0}\right)}{|x-y|^{3}}
$$

where we set for convenience $x=(r, 0,0)$. At $x=0$ the eigenfunctions are known. They behave for $y \sim 0$ nonsingular, i.e. the converge to a finite constant (zero included) for $y \rightarrow 0$. For large $y$ they decay exponentially. An explicite calculation shows $\mu^{\prime}(0)$ to be finite, hence by symmetry zero.

There are many questions even in this simple case still to be analyzed. So one expects the lowest eigenvalue $\mu_{0}(r)$ to be monotonically increasing in $r$ and not to cross any other eigenvalue. We will come back to these problems in our analysis of spectral projectors of electronic Hamiltonians.

Acknowledgements. It is our pleasure to thank J. M. Combes, A. Grossmann, R. Schrader, D. Uhlenbrock and D. Williams for many helpful discussions.

\section{Appendix A}

The aim of this appendix is to prove Theorem A which is used several times in the main part of the article. As a preparation we state the following

Lemma A. Let $T_{n}$ be a sequence of bounded linear operators on a Banach space $X$, converging strongly to $T$. Then $T_{n}$ converges uniformly on any compact set in $X$. 
This is a consequence of the principle of uniform boundedness. For a proof we refer to $[4$, p. 151]. Now we are ready to give a simple proof of

Theorem A. Let $\left\{T_{n}\right\}$ be a sequence of bounded linear operators, $n \in Z_{+}$, on a Banach space $X$ converging strongly to a linear operator $T$. Then the sequence $\left\{T_{n} K\right\}$ converges in norm to $T K$.

Proof. $K$ mapps the unit ball into a compact set $M \subset X$. On $M$ the $T_{n}$ 's converge uniformly i.e. for every $\varepsilon>0$ there exists a $N(\varepsilon)$ such that

$$
\left\|\left(T_{n}-T\right) K \phi\right\|<\varepsilon\|K \phi\| \forall \phi, \quad\|\phi\|=1, \quad n>N(\varepsilon) .
$$

But $K$ is bounded in the uniform topology hence $T_{n} K \stackrel{n}{\rightarrow} T K$.

Corollary A. Let $T_{n}$ be a sequence of linear bounded operators on a Hilbert space $Y$ such that $T_{n}^{*} \stackrel{s}{\rightarrow} T^{*}$. Let $K$ be compact. Then $K T_{n} \stackrel{n}{\rightarrow} K T$.

Proof. It is enough to prove norm convergence of $\left(K T_{n}\right)^{*}$ because $*$ is a norm continuous mapping of the bounded operators $B(x)$ onto $B(x)$. Yet $\left(K T_{n}\right)^{*}=T_{n}^{*} K^{*}$ and since $K^{*}$ is compact together with $K$ Theorem A can be applied.

\section{Appendix B}

The purpose of this appendix is to give a proof of Lemma 3. This will be done in several steps. First we show

Lemma B1. Let $z \in \mathbb{C}$ be in $\varrho(H(x)) \cap \varrho\left(H_{1}(\infty)\right)$ for $x$ in a neighborhood $U$ of infinity. Then

$$
\begin{aligned}
(z-H(x))^{-1}-\left(z-H_{1}(\infty)\right)^{-1}= & (z-H(x))^{-1} T(x) V_{2} T(-x)\left(z-H_{0}\right)^{-1} \\
& +(z-H(x))^{-1} B(x, z)
\end{aligned}
$$

and $B(x, z) \stackrel{n}{\rightarrow} 0$ for $|x| \rightarrow \infty$ and $z$ fixed.

Proof. Application of the resolvent equation shows B to be defined as follows:

$$
B(x, z)=T(x) V_{2} T(-x)\left(z-H_{0}\right)^{-1} V_{1}\left(z-H_{1}(\infty)\right)^{-1} .
$$

It was shown in the second paragraphe of the proof of Theorem 3 that $T(x) V_{2} T(-x)\left(z-H_{0}\right)^{-1} \rightarrow 0$ for $|x| \rightarrow \infty$. Since $V_{1}$ is $H_{0}$ compact and therefore $H_{1}(\infty)$ compact too, the statement follows from the corollary A.

Next we want to get some more information on the first term of the right hand side of (B1). This is given in

Lemma B2. Assume again $z \in \varrho(H(x)) \cap \varrho\left(H_{2}(\infty)\right)$ for $x \in U$. Then

$$
\begin{aligned}
(z-H(x))^{-1} T(x) V_{2} T(-x)\left(z-H_{0}\right)^{-1}= & T(x)\left(z-H_{2}(\infty)\right)^{-1} V_{2} T(-x)\left(z-H_{0}\right)^{-1} \\
& +C(x, z)
\end{aligned}
$$

and $C(x, z) \stackrel{n}{\rightarrow} 0$ for $|x| \rightarrow \infty$ and $z$ fixed.

Proof. A straightforward computation gives for $C$ the expression

$$
T(x)\left\{\left(z-H_{0}-T(-x) V_{1} T(x)-V_{2}\right)^{-1}-\left(z-H_{2}(\infty)\right)^{-1}\right\} V_{2}\left(z-H_{0}\right)^{-1} T(-x) \text {. }
$$


According to Theorem 3 the bracket converges strongly to zero. Since $V_{2}\left(z-H_{0}\right)^{-1}$ is compact by hypothesis, $C$ converges to zero in norm (Theorem A).

The next statement puts even more control on $C$ in the limit $|x| \rightarrow \infty$ :

Lemma B3. Under the same assumption as in the previous lemma one concludes

$$
C(x, z)=(z-H(x))^{-1} D(x, z)
$$

and $D(x, z)=V_{1} T(x)\left(z-H_{2}(\infty)\right)^{-1} V_{2}\left(z-H_{0}\right)^{-1} T(-x) \stackrel{n}{\rightarrow} 0$ for $|x| \rightarrow \infty, z$ fixed.

Proof. From (B.4) one gets for $D^{\prime}=T(-x) D T(x)$ the expression

$$
D^{\prime}=\left\{1-\left(z-H_{0}-T(-x) V_{1} T(x)-V_{2}\right)\left(z-H_{2}(\infty)\right)^{-1}\right\} V_{2}\left(z-H_{0}\right)^{-1}
$$

which is equal to

$$
D^{\prime}=T(-x) V_{1} T(x)\left(z-H_{2}(\infty)\right)^{-1} V_{2}\left(z-H_{0}\right)^{-1} .
$$

Yet the term $T(-x) V_{1} T(x)\left(z-H_{2}(\infty)\right)^{-1}$ converges strongly to zero for $|x| \rightarrow \infty$ because for every $u$ there exists a $v$ such that

$$
\left(z-H_{2}(\infty)\right)^{-1} u=\left(z-H_{0}\right)^{-1} .
$$

Now the assertion follows again from the second paragraphe of the proof of Theorem 3. Since $V_{2}\left(z-H_{0}\right)^{-1}$ is compact

$$
D^{\prime}(x, z) \stackrel{n}{\rightarrow} 0 \quad \text { for } \quad|x| \rightarrow \infty \quad \text { (Corollary A). }
$$

Summing up the information we get so far on the right hand side of (B.1) we get the equation

$$
\begin{aligned}
(z-H(x))^{-1}-\left(z-H_{1}(\infty)\right)^{-1}= & T(x)\left(z-H_{2}(\infty)\right)^{-1} V_{2} T(-x)\left(z-H_{0}\right)^{-1} \\
& +(z-H(x))^{-1}\{B(x, z)+D(x, z)\} .
\end{aligned}
$$

The operators $B$ and $D$ both converge in norm to zero for $|x| \rightarrow \infty$. The next lemma gives more information on how these operators converge to zero:

Lemma B4. Consider $\varepsilon>0, K \subset \mathbb{C}$ compact such that distance $d\left(K, \sigma\left(H_{l}(\infty)\right)\right)>\varepsilon$ for $l=1,2$ and $z \in \varrho(H(x)) \cap \varrho\left(H_{1}(\infty)\right) \cap \varrho\left(H_{2}(\infty)\right)$ for $x$ in a neighborhood $U$ of infinity. Then there exist for every $\eta>0$ a $\delta(\eta, K)$ such that $\|B(x, z)\|+\|D(x, z)\|<\eta$ for every $x$ with $\delta \mid x\}>1$. for $B$

Proof. 1. There exists a $\delta>0$ such that $\|B(x, z)\|<\frac{1}{2} \eta$ : From (B.2) one gets

$$
B=T(x) V_{2} T(-x)\left(z-H_{0}\right)^{-1} V_{1}\left(z-H_{0}\right)^{-1}\left\{1+V_{1}\left(z-H_{1}(\infty)\right)^{-1}\right\} .
$$

The bracket can be estimated in norm for every $z \in K$. But since the resolvent $\left(z-H_{1}(\infty)\right)^{-1}$ is analytic in $z$, the bound can be chosen uniformly for $z \in K$,

$$
\left\|1+V_{1}\left(z-H_{1}(\infty)\right)^{-1}\right\|<M, \forall z \in K .
$$

Yet the remaining part of $B$ converges in norm to zero for every $z \in K$. By the same argument as above there exists a $\delta(\eta, K)$ such that

$$
\left\|T(x) V_{2} T(-x)\left(z-H_{0}\right)^{-1} V_{1}\left(z-H_{0}\right)^{-1}\right\|<\frac{1}{2} \eta M^{-1}
$$

uniformly in $z$ and for $|x| \delta>1$. 
2. There exists a $\delta$ such that $\|D(x, z)\|<\frac{1}{2} \eta$ : Of course it is equivalent to show the same estimate for $D^{\prime}(x, z)=T(-x) D T(x)$. Changing the order of the terms in (B.5) yields

$$
D^{\prime}(x, z)=T(-x) V_{1} T(x)\left(z-H_{0}\right)^{-1} V_{2}\left(z-H_{2}(\infty)\right)^{-1} .
$$

Yet changing $x, V_{1}, V_{2}, H_{2}(\infty)$ into $-x, V_{2}, V_{1}, H_{1}(\infty)$ transforms $D^{\prime}(x, z)$ into $B(x, z)$ as defined in (B.2). Now the same argument as in the first paragraphe applies.

The statements of this appendix are summerized in Lemma 3 of the first chapter. There we used the abreviation $E=B+D$.

\title{
References
}

1. Born, M., Oppenheimer, R.: Zur Quantentheorie der Molekeln. Ann. Phys. 84, 457 (1927)

2. Combes, J.M., Duclos, P.: On the harmonic approximation (to be published)

Duclos, P.: On the Harmonic approximation. Thèse du 3-ème cycle, Faculté des sciences, Université de Luminy, Marseille 1974

3. Aventini, P., Combes, J.M., Duclos, P., Grossmann, A., Seiler, R.: On the method of Born and Oppenheimer (to be published)

Aventini,P.: On the method of Born and Oppenheimer. Thèse du 3-ème cycle, Faculté des sciences, Université de St. Charles, Marseille 1974

4. Kato, T.: Perturbation theory of linear operators. Berlin-Heidelberg-New York: Springer 1966

5. Balslev, E., Combes, J. M.: Spectral properties of many-body Schrödinger operators with dilationanalytic interactions. Commun. math. Phys. 22, 280 (1971)

6. Bates, D.R., Ledsham, K., Stewart, A.L.: Wave functions of the hydrogen molecular ion. Phil. Trans. Roy. Soc. London 246, 215 (1953)

Slater,J.C.: Quantum theory of molecules and solids, Vol. 1, Chapter 1. New York: McGraw-Hill Book Company 1963

7. Combes, J.M.: Relatively compact interactions in many partıcle systems. Commun. math. Phys. 12, 283 (1969)

8. Reed,M., Simon,B.: Methods of modern mathematical physics Vol. 1. New York: Academic Press 1972

9. Hunziker, W.: On the spectra of Schrödinger multi-particle Hamiltonians. Helv. Phys. Acta 39, 451 (1966)

10. Grossmann, A.: Complex canonical transformations and spectral theory; Proceedings of the $2^{\text {nd }}$ International Colloquium on Group Theoretical Methods in Physics, University of Nijmegen, The Netherlands, A 78, (1973)

Communicated by W. Hunziker

\author{
P. Aventini \\ R. Seiler \\ Institut für Theoretische Physik \\ Freie Universität Berlin \\ D-1000 Berlin 33 \\ Arnimallee 3
}

